\title{
Tutte chromatic identities from the Temperley-Lieb algebra
}

\author{
PAUL FENDLEY \\ VYACHESLAV KRUSHKAL
}

\begin{abstract}
This paper introduces a conceptual framework, in the context of quantum topology and the algebras underlying it, for analyzing relations obeyed by the chromatic polynomial $\chi(Q)$ of planar graphs. Using it we give new proofs and substantially extend a number of classical results concerning the combinatorics of the chromatic polynomial. In particular, we show that Tutte's golden identity is a consequence of level-rank duality for $\mathrm{SO}(N)$ topological quantum field theories and BirmanMurakami-Wenzl algebras. This identity is a remarkable feature of the chromatic polynomial relating $\chi(\phi+2)$ for any triangulation of the sphere to $(\chi(\phi+1))^{2}$ for the same graph, where $\phi$ denotes the golden ratio. The new viewpoint presented here explains that Tutte's identity is special to these values of the parameter $Q$. A natural context for analyzing such properties of the chromatic polynomial is provided by the chromatic algebra, whose Markov trace is the chromatic polynomial of an associated graph. We use it to show that another identity of Tutte's for the chromatic polynomial at $Q=\phi+1$ arises from a Jones-Wenzl projector in the Temperley-Lieb algebra. We generalize this identity to each value $Q=2+2 \cos (2 \pi j /(n+1))$ for $j<n$ positive integers. When $j=1$, these $Q$ are the Beraha numbers, where the existence of such identities was conjectured by Tutte. We present a recursive formula for this sequence of chromatic polynomial relations.
\end{abstract}

57M15; 05C15, 57R56, 81R05

\section{Introduction}

In a series of papers in 1970 [23; 22], W T Tutte discovered several remarkable properties of the chromatic polynomial $\chi(Q)$ of planar graphs evaluated at the special value $Q=\phi+1$, where $\phi$ denotes the golden ratio, $\phi=(1+\sqrt{5}) / 2$. Among these is the "golden identity": for a planar triangulation $T$,

$$
\chi_{T}(\phi+2)=(\phi+2) \phi^{3 V(T)-10}\left(\chi_{T}(\phi+1)\right)^{2},
$$

where $V(T)$ is the number of vertices of the triangulation. Tutte used this identity to establish that $\chi_{T}(\phi+2)$ is positive, a result interesting in connection to the four-color 
theorem. Another property (see (11.15) in [24]) is the relation

$$
\chi_{Z_{1}}(\phi+1)+\chi_{Z_{2}}(\phi+1)=\phi^{-3}\left(\chi_{Y_{1}}(\phi+1)+\chi_{Y_{2}}(\phi+1)\right),
$$

where $Y_{i}, Z_{i}$ are planar graphs which are locally related as shown in Figure 1.
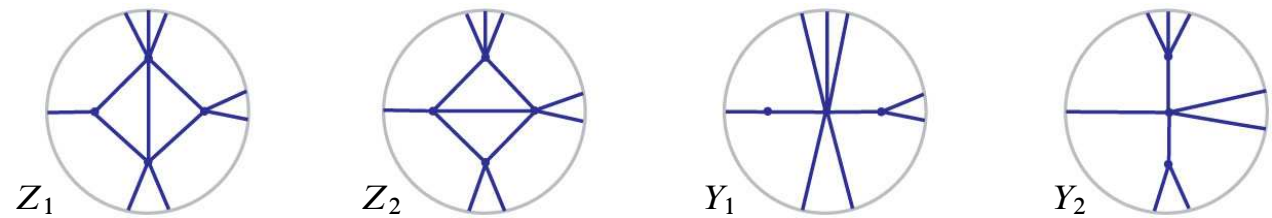

Figure 1: The graphs in Tutte's identity (1-2). There can be any number of lines from each vertex to the boundary of the disk, and the graphs are identical outside the disk.

The main purpose of this paper is to show that Tutte's results naturally fit in the framework of quantum topology and the algebras underlying it. We give conceptual proofs of these identities, very different from the combinatorial proofs of Tutte's. This allows us to show that both identities (1-1) and (1-2) are not ad hoc, but are (particularly elegant) consequences of a deeper structure. We generalize the linear relation (1-2) to other special values of $Q$, dense in the interval $[0,4]$.

For example, the identity analogous to (1-2) at $Q=2$ is simply that $\chi_{\Gamma}(2)=0$ whenever the graph $\Gamma$ includes a triangle. This vanishing is completely obvious from the original definition of $\chi_{\Gamma}(Q)$ for integer $Q$ as the number of $Q$-colorings of $\Gamma$ such that adjacent vertices are colored differently. Nevertheless, this vanishing does not follow immediately from the contraction-deletion relation used to define $\chi(Q)$ for all $Q$. We use the algebraic approach to fit this obvious identity and the rather nontrivial identity (1-2) into a series of identities at special values of $Q$ which are independent of the contraction-deletion relation, although of course are consistent with it.

We show that Tutte's golden identity (1-1) is a consequence of level-rank duality for $\mathrm{SO}(N)$ TQFTs (or equivalently $U_{q}(\operatorname{so}(N))$ representation theory) and the BirmanMurakami-Wenzl algebras underlying the construction of the (doubled) TQFTs. Levelrank duality is an important property of conformal field theories and topological quantum field theories. It implies that the $\mathrm{SO}(N)$ level $k$, and the $\mathrm{SO}(k)$ level $N$ theories are isomorphic; see Mlawer et al [17] (in our conventions the level $k$ of $\mathrm{SO}(3)$ corresponds to the level $2 k$ of $\mathrm{SU}(2)$, so the $\mathrm{SO}(3)$ theories are labeled by the half-integers). We exploit the isomorphism between the $\mathrm{SO}(3)_{4}$ and $\mathrm{SO}(4)_{3}$ theories, and then show that the latter splits into a product of two copies of $\mathrm{SO}(3)_{3 / 2}$. The partition function of an $\mathrm{SO}(3)$ theory is given in terms of the chromatic polynomial, specifically $\chi(\phi+2)$ for 
$\mathrm{SO}(3)_{4}$ and $\chi(\phi+1)$ for $\mathrm{SO}(3)_{3 / 2}$. These isomorphisms enable us to give a rigorous proof of the golden identity (1-1). This new viewpoint makes it clear that the fact that golden identity relates a chromatic polynomial squared to the chromatic polynomial at another value is very special to these values of $Q$ : generalizations using level-rank duality $\mathrm{SO}(N)_{4} \leftrightarrow \mathrm{SO}(4)_{N}$ do not involve the chromatic polynomial.

An important and rather natural tool for analyzing the chromatic polynomial relations is the chromatic algebra $\mathcal{C}_{n}^{Q}$ introduced (in a different context) by Martin and Woodcock [16], and analyzed in depth by the authors in [6]. The basic idea in the definition of the chromatic algebra is to consider the contraction-deletion rule as a linear relation in the vector space spanned by graphs, rather than just a relation defining the chromatic polynomial. In this context, the right object to study is the space of dual graphs, and the algebra is defined so that the Markov trace of a graph is the chromatic polynomial of its dual. The parameter $Q$ in the chromatic algebra $\mathcal{C}^{Q}$ is related to the value of the loop (or dually the value of the chromatic polynomial of a single point). Several authors have considered similar algebraic constructions, for example Jones [11] in the context of planar algebras, Kuperberg [15] in the rank 2 case, Martin and Woodcock [16] for deformations of Schur algebras and Walker $[25 ; 26]$ in the TQFT setting. In fact, the relation between $\mathrm{SO}(3)$ representation theory and colorings of planar graphs dates back to spin networks of Penrose [19]. We use the chromatic algebra to systematically analyze the structure of the chromatic polynomial of planar graphs.

Relations between algebras and topological invariants like the chromatic polynomial are familiar from both knot theory and statistical mechanics. Our companion paper [6] discusses these connections in detail. In particular, the relation between the $\mathrm{SO}(3)$ Birman-Wenzl-Murakami algebra and the chromatic algebra described initially by the first author and Read [7] is derived there. This in turn yields a relation between the chromatic algebra and the Temperley-Lieb algebra [7], which is described in Section 2. Readers familiar with the Potts model and/or the Tutte polynomial may recall that it has long been known (indeed from Temperley and Lieb's original paper [21]) that the chromatic polynomial and the more general Tutte polynomial can be related to the Markov trace of elements of the Temperley-Lieb algebra. The relation derived in [7] and here is quite different from the earlier relation; in statistical-mechanics language ours arises from the low-temperature expansion of the Potts model, while the earlier one arises from Fortuin-Kasteleyn cluster expansion [6].

The relation of the chromatic algebra to the Temperley-Lieb algebra utilized here makes it possible to rederive and greatly extend Tutte's identities. In this context, linear identities such as Tutte's (1-2) are understood as finding elements of the trace radical: elements of the chromatic algebra which, multiplied by any other element of the algebra, are in the kernel of the Markov trace. For example, it is well known how 
the Jones-Wenzl projectors $P^{(k)}$, crucial for the construction of SU(2) topological quantum field theories, are defined within the Temperley-Lieb algebra. We define these relations in the chromatic algebra, for special values of the parameter $Q$, as a pullback of the Jones-Wenzl projectors. Tutte's relation (1-2) is a pullback of the projector $P^{(4)}$, corresponding to $Q=\phi+1$. More precisely, in this algebraic setting the relation (1-2) for the chromatic polynomials of the graphs in Figure 1 is a consequence of the fact that

$$
\widehat{Z}_{1}+\widehat{Z}_{2}-\phi^{-3}\left(\widehat{Y}_{1}+\widehat{Y}_{2}\right)
$$

is an element of the trace radical of the algebra $\mathcal{C}_{2}^{\phi+1}$. Here $\widehat{Y}_{i}, \widehat{Z}_{i}$ are the planar graphs dual to the graphs $Y_{i}, Z_{i}$; see Figure 2.
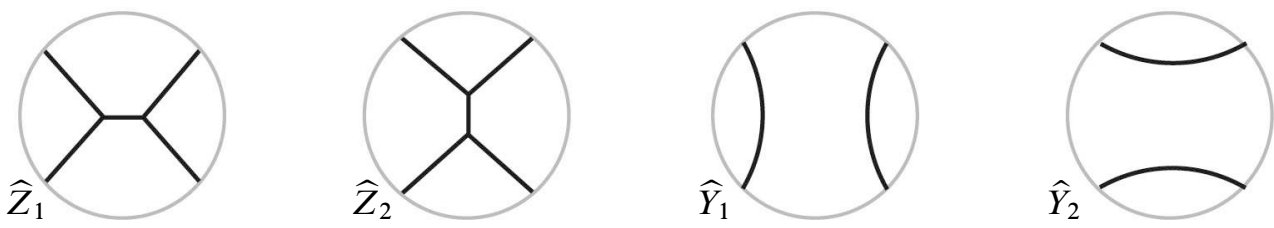

Figure 2: The graphs $\widehat{Y}_{i}, \widehat{Z}_{i}$ dual to the graphs in Figure 1

Tutte remarks about (1-2): "This equation can be taken as the basic one in the theory of golden chromials" [24]. This statement has a precise meaning in our algebraic context: $P^{(4)}$ is a generator of the unique proper ideal of the Temperley-Lieb category related to the chromatic polynomial at $Q=\phi+1$. Note that Tutte's discovery of this relation (for this specific value of $Q$ ) predates that of Jones and Wenzl by fifteen years!

We show that Tutte's identity (1-2) has an analog for any value of $Q$ obeying

$$
Q=2+2 \cos \left(\frac{2 \pi j}{n+1}\right)
$$

for $j$ and $n$ positive integers obeying $j<n$. The requirement of integer $n$ arises from the structure of the Temperley-Lieb category: these are the only values for which the Temperley-Lieb category has a nontrivial proper ideal (given by the trace radical). When $j=1$, these values of $Q$ are the Beraha numbers $B_{n+1}$, discussed in more detail below. Tutte conjectured in [24] that there is such a relation, similar to (1-2) at $\phi+1=B_{5}$, for each Beraha number, and we give a recursive formula for these relations based on the formula for the Jones-Wenzl projectors $P^{(n)}$. In fact, based on the relation between the chromatic algebra and the $\mathrm{SO}(3) \mathrm{BMW}$ algebra [6], it seems reasonable to conjecture that these are all linear relations which preserve the chromatic 
polynomial at a given value of $Q$ (and in particular there are no such relations at $Q$ not equal to one of special values (1-4)).

We use similar ideas to give also a direct algebraic proof of the golden identity (1-1). This proof (given in Section 3) is motivated by the argument using level-rank duality, discussed above, but it is given entirely in the context of the chromatic algebra. It is based on a map $\Psi: \mathcal{C}^{\phi+2} \longrightarrow\left(\mathcal{C}^{\phi+1} / R\right) \otimes\left(\mathcal{C}^{\phi+1} / R\right)$, where $R$ denotes the trace radical. We show that the existence of this map is implied by the relation (1-2), and then (1-1) follows from applying the algebra traces to the homomorphism $\Psi$. The golden identity has an interesting application in physics in quantum loop models of "Fibonacci anyons", where it implies that these loop models should yield topological quantum field theories in the continuum limit (see Fendley [5], Fidkowski et al [8] and Walker [26] for more details).

We conclude this general discussion of Tutte's results by noting his estimate:

$$
\left|\chi_{T}(\phi+1)\right| \leq \phi^{5-k},
$$

where $T$ is a planar triangulation and $k$ is the number of its vertices. The origin of Beraha's definition of the numbers $B_{n}$ is his observation [2] that the zeros of the chromatic polynomial of large planar triangulations seem to accumulate near them. Tutte's estimate (1-5) gives a hint about this phenomenon for $B_{5}$. Efforts have been made (see Kauffman and Saleur [14] and Saleur [20]) to explain Beraha's observation using quantum groups. We hope that our approach will shed new light on this question. The paper is organized as follows. Section 2 introduces the chromatic algebra $\mathcal{C}_{n}^{Q}$, reviews the standard material on the Temperley-Lieb algebra $\mathrm{TL}_{n}^{d}$, and describes the algebra homomorphism $\mathcal{C}_{n}^{Q} \longrightarrow \mathrm{TL}_{2 n}^{\sqrt{Q}}$. It then shows that Tutte's relation (1-2) is the pullback of the Jones-Wenzl projector $P^{(4)} \in \mathrm{TL}^{\phi}$. With this relation at hand, Section 3 gives a direct algebraic proof of Tutte's golden identity (1-1) in the chromatic algebra setting. Section 4 discusses the level-rank duality of $\mathrm{SO}(N) \mathrm{BMW}$ algebras, and shows that the golden identity (1-1) is its consequence. In the final Section 5, we show that for any $Q$ obeying (1-4), the chromatic polynomial obeys a generalization of (1-2). We give a recursive formula for this sequence of chromatic relations. This paper is largely self-contained, but we refer the reader interested in the chromatic algebra, the $\mathrm{SO}(3)$ Birman-Murakami-Wenzl algebra and the relations between the chromatic polynomial, link invariants and TQFTs, to our companion paper [6].

\section{The chromatic algebra and the Temperley-Lieb algebra}

The definition of the algebraic structure of the chromatic algebra $\mathcal{C}_{n}^{Q}$ - the product structure, the trace, the inner product - is motivated by that of the Temperley-Lieb 
algebra. In this section we set up the general framework for relating the chromatic polynomial to the Temperley-Lieb algebra, and we show that the relation (1-2) corresponds to a Jones-Wenzl projector. We start by defining the chromatic algebra [16;6], and then review standard material on Temperley-Lieb algebra (the reader is referred to Kauffman and Lins [13] for more details). We then define an algebra homomorphism $\mathcal{C}_{n}^{Q} \longrightarrow \mathrm{TL}_{2 n}^{d}$, where $Q=d^{2}$, which respects these structures. In particular, the pullback under this homomorphism of the trace radical in $\mathrm{TL}_{2 n}^{d}$ (the ideal consisting of elements whose inner product with all other elements in the algebra is trivial) is in the trace radical of $\mathcal{C}_{2 n}^{Q}$, which corresponds to local relations on graphs which preserve the chromatic polynomial of the duals, for a given value of $Q$. In fact, the relevant algebraic structure here is the chromatic, respectively Temperley-Lieb, category.

\subsection{The chromatic algebra}

The chromatic polynomial $\chi_{\Gamma}(Q)$ of a graph $\Gamma$, for $Q \in \mathbb{Z}_{+}$, is the number of colorings of the vertices of $\Gamma$ with the colors $1, \ldots, Q$ where no two adjacent vertices have the same color. A basic property of the chromatic polynomial is the contraction-deletion rule: given any edge $e$ of $\Gamma$ which is not a loop,

$$
\chi_{\Gamma}(Q)=\chi_{\Gamma \backslash e}(Q)-\chi_{\Gamma / e}(Q)
$$

where $\Gamma \backslash e$ is the graph obtained from $\Gamma$ by deleting $e$, and $\Gamma / e$ is obtained from $\Gamma$ by contracting $e$. If $\Gamma$ contains a loop then $\chi_{\Gamma} \equiv 0$; if $\Gamma$ has no edges and $V$ vertices, then $\chi_{\Gamma}(Q)=Q^{V}$. These properties enable one to define $\chi_{\Gamma}(Q)$ for any, not necessarily integer, values of $Q$.

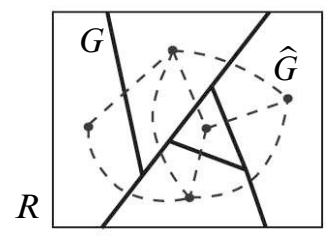

Figure 3: A basis element $G$ of the algebra $\mathcal{F}_{2}$ and the dual graph $\widehat{G}$ (drawn dashed)

Consider the free algebra $\mathcal{F}_{n}$ over $\mathbb{C}[Q]$ whose elements are formal linear combinations of the isotopy classes of planar trivalent graphs in a rectangle $R$ (Figure 3 ). The intersection of each such graph with the boundary of $R$ consists of precisely $2 n$ points: $n$ points at the top and the bottom each, and the isotopy, defining equivalent graphs, is required to preserve the boundary. Note that the vertices of the graphs in the interior of $G$ are trivalent, in particular they do not have ends (1-valent vertices) other than 
those on the boundary of $R$. It is convenient to allow 2-valent vertices as well, so there may be loops disjoint from the rest of the graph. The multiplication in $\mathcal{F}_{n}$ is given by vertical stacking, and the inclusion of algebras $\mathcal{F}_{n} \subset \mathcal{F}_{n+1}$ is defined on the graphs generating $\mathcal{F}_{n}$ as the addition of a vertical strand on the right. Given $G \in \mathcal{F}_{n}$, the vertices of its dual graph $\widehat{G}$ correspond to the complementary regions $R \backslash G$, and two vertices are joined by an edge in $\widehat{G}$ if and only if the corresponding regions share an edge, as illustrated in Figure 3.

Definition 2.1 The chromatic algebra in degree $n, \mathcal{C}_{n}$, is the algebra over $\mathbb{C}[Q]$ which is defined as the quotient of the free algebra $\mathcal{F}_{n}$ by the ideal $I_{n}$ generated by the relations shown in Figure 4. In addition, the value of a trivial simple closed curve is set to be $Q-1$. When the parameter $Q$ is specialized to a complex number, the resulting algebra over $\mathbb{C}$ is denoted $\mathcal{C}_{n}^{Q}$. Set $\mathcal{C}=\cup_{n} \mathcal{C}_{n}$.

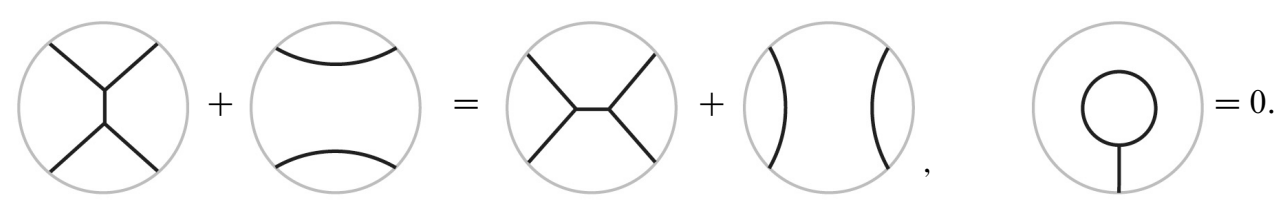

Figure 4: Relations in the trivalent presentation of the chromatic algebra

The first relation in Figure 4 is sometimes known as the "H-I relation", while the second is requiring that "tadpoles" vanish. A few words may be helpful in explaining the relations defining the chromatic algebra. If there is a simple closed curve bounding a disk in a rectangular picture, disjoint from the rest of the graph, it may be erased while the element represented in $\mathcal{C}_{n}$ is multiplied by $Q-1$. The ideal corresponding to the relation on the left in Figure 4 is generated by linear combinations of graphs in $\mathcal{F}_{n}$ which are identical outside a disk in the rectangle, and which differ according to this relation in the disk, Figure 5. This may be naturally expressed in the language of planar algebras (see also Section 5).

Remark In this definition we used just the trivalent graphs, and this is sufficient for the purposes of this section, in particular for the proofs of Tutte's identities (1-1), (1-2). The definition of the chromatic algebra using all planar graphs (isomorphic to the trivalent one considered here), is given in Section 5.

Definition 2.2 The trace, $\operatorname{tr}_{\chi}: \mathcal{C} Q \longrightarrow \mathbb{C}$ is defined on the additive generators (graphs) $G$ by connecting the endpoints of $G$ by disjoint arcs in the complement of the rectangle 


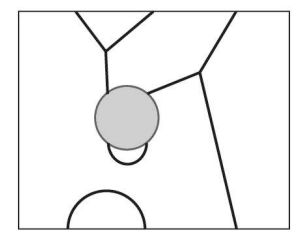

Figure 5: An element in the ideal $I_{3}$. The shaded disk contains the relation on the left in Figure 4.

$R$ in the plane (denote the result by $\bar{G}$ ) and evaluating

$$
\operatorname{tr}_{\chi}(G)=Q^{-1} \cdot \chi \widehat{\bar{G}}(Q)
$$

Figure 6 shows the trace of an example. The factor $Q^{-1}$ provides a normalization of

$\operatorname{tr}$

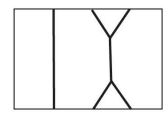

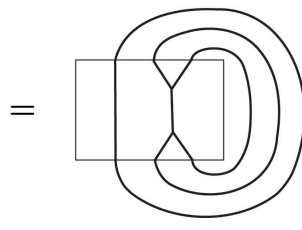

Figure 6: An example of the evaluation of the trace

the trace which turns out to be convenient from the point of view of the relation with the Temperley-Lieb algebra (see below). One checks that the trace is well-defined by considering the chromatic polynomial of the dual graphs. Specifically, the second relation in Figure 4 holds since the dual graph has a loop. The first relation holds since (in the notation in Figure 1), the deletion-contraction rule for the chromatic polynomial implies

$$
\chi_{Z_{1}}(Q)+\chi_{Y_{1}}(Q)=\chi_{Z_{2}}(Q)+\chi_{Y_{2}}(Q)
$$

Finally, the relation replacing a trivial simple closed curve by a factor $(Q-1)$ corresponds to the effect on the chromatic polynomial of the dual graph of erasing a 1-valent vertex and of the adjacent edge. 


\subsection{The Temperley-Lieb algebra}

The Temperley-Lieb algebra in degree $n, \mathrm{TL}_{n}$, is an algebra over $\mathbb{C}[d]$ generated by $1, E_{1}, \ldots, E_{n-1}$ with the relations [21]

$$
E_{i}^{2}=E_{i}, \quad E_{i} E_{i \pm 1} E_{i}=\frac{1}{d^{2}} E_{i}, \quad E_{i} E_{j}=E_{j} E_{i} \text { for }|i-j|>1 .
$$

Define $\mathrm{TL}=\bigcup_{n} \mathrm{TL}_{n}$. The indeterminate $d$ may be set to equal a specific complex number, and when necessary, we will include this in the notation, $\mathrm{TL}_{n}^{d}$.

It is convenient to represent the elements of $\mathrm{TL}_{n}$ pictorially: in this setting, an element of $\mathrm{TL}_{n}$ is a linear combination of 1 -dimensional submanifolds in a rectangle $R$. Each submanifold meets both the top and the bottom of the rectangle in exactly $n$ points. The multiplication then corresponds to vertical stacking of rectangles. The generators of $\mathrm{TL}_{3}$ are illustrated in Figure 7.
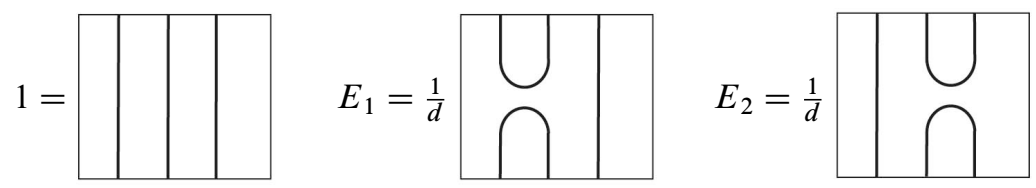

Figure 7: Generators of $\mathrm{TL}_{3}$

The rectangular pictures are considered equivalent if they are isotopic relative to the boundary. Another equivalence arises from the relation $E_{i}^{2}=E_{i}$ : the element in TL corresponding to a picture in $R$ with a circle (a simple closed curve) is equivalent to the element with the circle deleted and multiplied by $d$. Isotopy together with this relation are sometimes referred to as $d$-isotopy [9].

The trace $\operatorname{tr}_{d}: \mathrm{TL}_{n}^{d} \longrightarrow \mathbb{C}$ is defined on the additive generators (rectangular pictures) by connecting the top and bottom endpoints by disjoint arcs in the complement of $R$ in the plane (the result is a disjoint collection of circles in the plane), and then evaluating $d^{\# \text { circles }}$. The Hermitian product on $\mathrm{TL}_{n}$ is defined by $\langle a, b\rangle=\operatorname{tr}(a \bar{b})$, where the involution $\bar{b}$ is defined by conjugating the complex coefficients, and on an additive generator $b$ (a picture in $R$ ) is defined as the reflection in a horizontal line.

\subsection{A map from the chromatic algebra to the Temperley-Lieb algebra}

Definition 2.3 Define a homomorphism $\Phi: \mathcal{F}_{n} \longrightarrow \mathrm{TL}_{2 n}^{d}$ on the multiplicative generators (trivalent graphs in a rectangle) of the free algebra $\mathcal{F}_{n}$ by replacing each edge 
with the linear combination $\Phi(\mid)=)\left(-\frac{1}{d} \asymp\right.$, and resolving each vertex as shown in Figure 8. Moreover, for a graph $G, \Phi(G)$ contains a factor $d^{V(G) / 2}$, where $V(G)$ is the number of vertices of $G$.

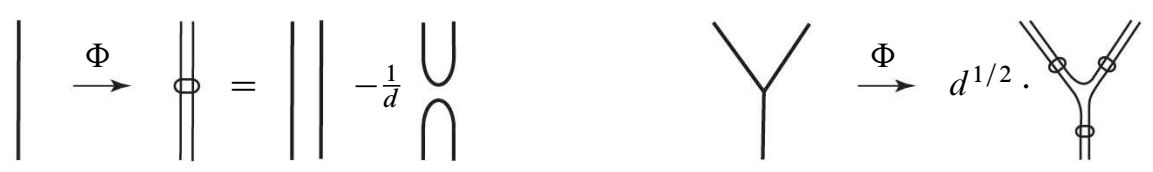

Figure 8: Definition of the homomorphism $\Phi: \mathcal{C}_{n}^{Q} \longrightarrow \mathrm{TL}_{2 n}^{\sqrt{Q}}$

Remarks (1) The reader may have noticed that $\Phi$ replaces each edge with the second Jones-Wenzl projector $P_{2}$, well-known in the study of the Temperley-Lieb algebra [27]. They are idempotents: $P_{2} \circ P_{2}=P_{2}$, and this identity (used below) may be easily checked directly; see Figure 9.

$$
\oiint=||-\frac{1}{d} \bigcap^{-\frac{1}{d}} \underset{\bigcap}{\bigcup}+\frac{1}{d^{2}} \underset{\bigcap}{\bigcap}=\$
$$

Figure 9: $P_{2} \circ P_{2}=P_{2}$

(2) Various authors have considered versions of the map $\Phi$ in the knot-theoretic and TQFT contexts; see Yamada [29], Jaeger [10], Kauffman and Lins [13], Fidkowski et al [8], Walker [26] and Fendley [5]. In [7], the first author and Read used this to give a map of the $\mathrm{SO}(3)$ BMW algebra to the Temperley-Lieb algebra, probably the first instance where this map is considered as an algebra homomorphism.

Lemma 2.4 $\Phi$ induces a well-defined homomorphism of algebras $\mathcal{C}_{n}^{Q} \longrightarrow \mathrm{TL}_{2 n}^{d}$, where $Q=d^{2}$.

Proof To prove this lemma, one needs to check that the relations in $\mathcal{C}_{n}^{Q}$, Figure 4, hold in the Temperley-Lieb algebra. It follows from the definition of $\Phi$ (Figure 8) that the relation in Figure 10 holds. Similarly, one checks the other two defining relations of the chromatic algebra.

The following lemma implies that the homomorphism $\Phi$ preserves the trace of the chromatic, respectively Temperley-Lieb, algebras (see also Theorem 1 in [8]). 


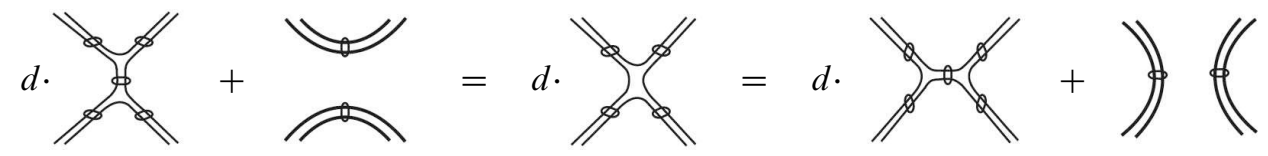

Figure 10

Lemma 2.5 Let $G$ be a trivalent planar graph. Then

$$
Q^{-1} \chi_{Q}(\widehat{G})=\Phi(G) .
$$

Here $Q=d^{2}$ and, abusing the notation, we denote by $\Phi(G)$ the evaluation $d^{\#}$ applied to the linear combination of simple closed curves obtained by applying $\Phi$ as shown in Figure 8. Therefore, the following diagram commutes:

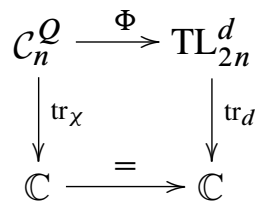

For example, for the theta-graph $G$ in Figure 11, one checks that

$$
Q^{-1} \chi_{Q}(\widehat{G})=(Q-1)(Q-2)=d^{4}-3 d^{2}+2=\Phi(G) .
$$
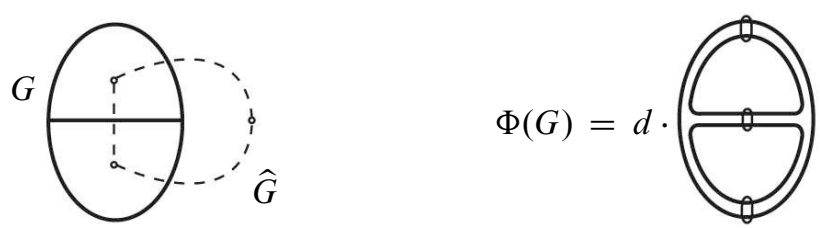

Figure 11

Proof of Lemma 2.5 We use the state sum formula for the chromatic polynomial (cf Bollobás [4]):

$$
\chi_{Q}(\widehat{G})=\sum_{s \subseteq E(\widehat{G})}(-1)^{|s|} Q^{k(s)} .
$$

Here $k(s)$ is the number of connected components of the graph $\widehat{G}_{S}$ whose vertex set is $V(\widehat{G})$ and the edge set is $s$. 
First assume that $G$ is a connected graph. Recall that $\Phi(G)$ is obtained from $G$ by replacing each edge $\mid$ by the linear combination ()$(-1 / d \asymp)$, and resolving each vertex as shown in Figure 8. Then $\Phi(G)$ is (the evaluation of) a linear combination of simple closed curves. This linear combination can be represented as a sum parametrized by the subsets of the set of the edges of the dual graph $\widehat{G}, s \subset E(\widehat{G})$. For each such subset, the corresponding term is obtained by replacing each edge $\mid$ of $G$ not intersecting $s$ with ) (, and each edge intersecting $s$ with $\asymp$. Moreover, this collection of simple closed curves is the boundary of a regular neighborhood of the graph $\widehat{G}_{s}$ (this is checked inductively, starting with the case $s=\varnothing$, and looking at the effect of adding one edge at a time). Therefore their number equals the number of connected components of the graph $\widehat{G}_{S}$, plus the rank of the first homology of $\widehat{G}_{S}$, denoted $n(S)$. Then

$$
\Phi(G)=d^{V(G) / 2} \sum_{s \subseteq E(\widehat{G})}(-1)^{|s|} \frac{1}{d^{|s|}} d^{k(s)+n(s)} .
$$

We claim that the corresponding terms in the expansions of $Q^{-1} \chi_{Q}(\widehat{G}), \Phi(G)$ are

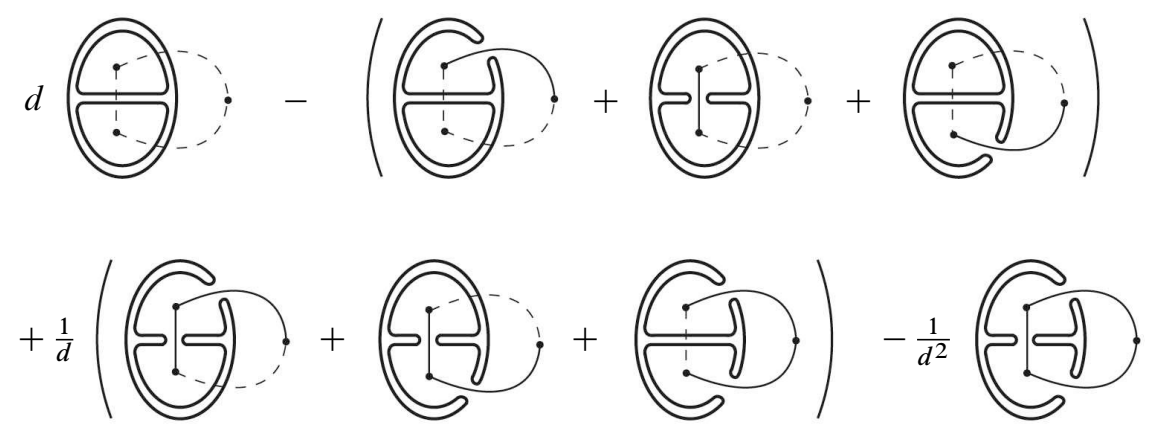

Figure 12: The expansions of $Q^{-1} \chi_{Q}(\widehat{G}), \Phi(G)$ where $G$ is the theta graph in Figure 11. The edges of $\widehat{G}$ in each term which are in the given subset $s$ are drawn solid, other edges are dashed.

equal. (See Figure 12 for the expansions of $Q^{-1} \chi_{Q}(\widehat{G}), \Phi(G)$ in the example $G=$ the theta graph shown in Figure 11.) Since $G$ is a trivalent graph and we assumed $G$ is connected, its dual $\widehat{G}$ is a triangulation (each face of $\widehat{G}$ has three edges), so that $2 V(\widehat{G})=F(\widehat{G})+4=V(G)+4$. Therefore

$$
\Phi(G)=\sum_{s \subseteq E(\widehat{G})}(-1)^{|s|} d^{V(\widehat{G})-2+k(s)+n(s)-|s|} .
$$


The exponent simplifies because $k(s)-n(s)+|s|=V(\widehat{G})$. This is obviously true for $s=\varnothing$, and inductively an addition of one edge to $s$ either decreases $k(s)$ by 1 , or it increases $n(s)$ by 1 , so $k(s)-n(s)+|s|$ remains equal to $V(\widehat{G})$. Because $Q=d^{2}$,

$$
Q^{-1} \chi_{Q}(\widehat{G})=\sum_{s \subseteq E(\widehat{G})}(-1)^{|s|} d^{2 k(s)-2}=\Phi(G) .
$$

This concludes the proof of Lemma 2.5 for a connected graph $G$.

Now let $G$ be a trivalent, not necessarily connected, planar graph. To be specific, first suppose $G$ has two connected components, $G=G_{1} \sqcup G_{2}$. It follows from the definition of $\Phi$ that $\Phi(G)=\Phi\left(G_{1}\right) \cdot \Phi\left(G_{2}\right)$. Also note that $\widehat{G}$ is obtained from $\widehat{G}_{1}$, $\widehat{G}_{2}$ by identifying a single vertex. It is a basic property of the chromatic polynomial that in this situation

$$
\chi_{\widehat{G}}(Q)=Q^{-1} \chi_{\widehat{G}_{1}}(Q) \cdot \chi_{\widehat{G}_{2}}(Q) .
$$

Since the equality (2-4) holds for connected graphs $G_{1}, G_{2}$, it also holds for $G$. This argument gives an inductive proof of (2-4) for trivalent graphs with an arbitrary number of connected components.

The trace radical of an algebra $A$ is the ideal consisting of the elements $a$ of $A$ such that $\operatorname{tr}(a b)=0$ for all $b \in A$. The local relations on graphs which preserve the chromatic polynomial of the dual, for a given value of $Q$, correspond to the elements of the trace radical of $A=\mathcal{C}_{n}^{Q}$ (see Section 5). It follows from Lemma 2.5 that the pullback by $\Phi$ of the trace radical in $\mathrm{TL}^{d}$ is in the trace radical of $\mathcal{C}^{d^{2}}$.

When $d=\phi$, the trace radical of the Temperley-Lieb algebra is generated by the Jones-Wenzl projector $P^{(4)}$ displayed in Figure 13. It is straightforward to check that $\Phi$ maps the element (1-3) to $P^{(4)}$. We have therefore established Tutte's relation (1-2) as a consequence of Lemma 2.5 and of the properties of the Jones-Wenzl projector. In Section 5 we derive a recursive formula for the identities of the chromatic polynomial at $Q$ obeying (1-4), which yields the identity (1-2) as a special case.

\section{Tutte's golden identity for the chromatic polynomial}

In this section we give a proof of Tutte's golden identity in the algebraic setting, established in the previous section. (Section 4 below presents an alternative proof using level-rank duality of the BMW algebras.)

Theorem 1 For a planar triangulation $\widehat{G}$,

$$
\chi_{\widehat{G}}(\phi+2)=(\phi+2) \phi^{3 V(\widehat{G})-10}\left(\chi_{\widehat{G}}(\phi+1)\right)^{2}
$$

where $V(\widehat{G})$ is the number of vertices of $\widehat{G}$. 


$$
\begin{aligned}
& \left.P^{(4)}=||\left|-\frac{d}{d^{2}-2}\right| \begin{array}{l}
\cup \\
\cap
\end{array}\left|+\frac{1}{d^{2}-2}(|/ / \cap| \bigcap\rangle / \cap\right|\right) \\
& +\frac{-d^{2}+1}{d^{3}-2 d}\left(\left|\begin{array}{c}
\cup \\
\cap
\end{array}\right| \quad\left|\begin{array}{c}
\cup \\
\cap
\end{array}\right|\right)-\frac{1}{d^{3}-2 d}\left(\begin{array}{lll}
\cup & \backslash \\
\cap & \cap
\end{array}\right)
\end{aligned}
$$

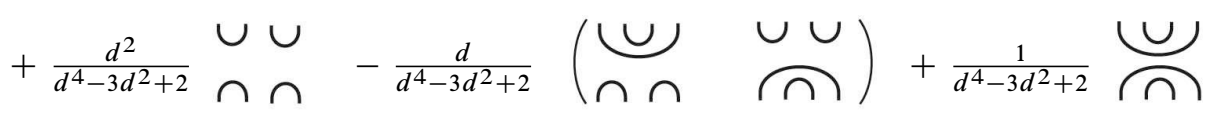

Figure 13: The Jones-Wenzl projector $P^{(4)}$ which generates the trace radical of $\mathrm{TL}^{\phi} . P^{(4)}$ is defined for all values of $d$, and to get an element of $\mathrm{TL}^{\phi}$ one sets $d=\phi$ in the formula above. The homomorphism $\Phi: \mathcal{C}^{\phi+1} \longrightarrow \mathrm{TL}^{\phi}$ maps the relation (1-3): $\widehat{Z}_{1}+\widehat{Z}_{2}=\phi^{-3}\left[\widehat{Y}_{1}+\widehat{Y}_{2}\right]$, dual to Tutte's identity $(1-2)$, to the relation $P^{(4)}=0$.

For the subsequent proof, it is convenient to reexpress this identity in terms of the graph $G$ dual to $\widehat{G}$. Since $\widehat{G}$ is a triangulation, $G$ is a connected trivalent graph. Using the Euler characteristic, one observes that the number of faces $F(\widehat{G})=2 V(\widehat{G})-4$. Since $V(G)=F(\widehat{G})$, the golden identity may be rewritten as

$$
\chi_{\widehat{G}}(\phi+2)=\frac{\phi+2}{\phi^{4}} \phi^{3 V(G) / 2}\left(\chi_{\widehat{G}}(\phi+1)\right)^{2} .
$$

Proof of Theorem 1 Consider the vector space $\mathcal{F} T$ over $\mathbb{C}$ spanned by all connected planar trivalent graphs. (There are no relations imposed among these graphs, so this is an infinite dimensional vector space.) Define a map $\Psi: \mathcal{F} T \longrightarrow \mathcal{F} T \otimes \mathcal{F} T$ on the generators by

$$
\Psi(G)=\phi^{3 V / 2}(G \otimes G),
$$

where $V$ is the number of vertices of a trivalent graph $G$. (See Figure 14 illustrating the cases $V=1,2$.) Here $\phi$ denotes the golden ratio.

Consider the map $\pi_{Q}: \mathcal{F} T \longrightarrow \mathbb{C}$ defined by taking the quotient of $\mathcal{F} T$ by the ideal generated by the relations in the trivalent presentation of the chromatic algebra, as given in Definition 2.1 and in Figure 4. This quotient is 1-dimensional because the graphs in $\mathcal{F} T$ have no ends; applying the relations allows any such graph to be reduced to a number. Namely, this projection map $\pi_{Q}$ applied to a graph is the quantum evaluation, or equivalently it is equal to $Q^{-1}$ times the chromatic polynomial of the dual graph. 

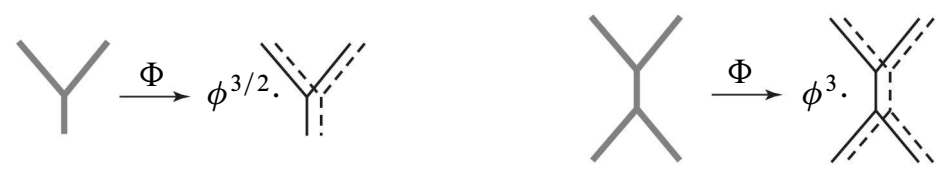

Figure 14: The map $\Psi: \mathcal{F} T \longrightarrow \mathcal{F} T \otimes \mathcal{F} T$. The different kinds of lines representing the graphs (gray, solid and dashed) correspond to the three different copies of $\mathcal{F} T$.

Tutte's identity follows from the following statement:

Lemma 3.1 The following diagram commutes:

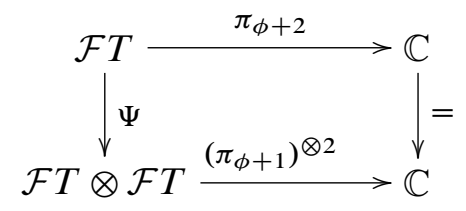

Proof The strategy is to check that the image under $\Psi$ of the three relations in $\mathcal{F} T$ given in Definition 2.1 and Figure 4 at $Q=\phi+2$, hold as a consequence of the relations in $\mathcal{F} T \otimes \mathcal{F} T$ at $Q=\phi+1$. Showing this means that $(1 /(\phi+2)) \chi_{\widehat{G}}(\phi+2)$ for any $G$ in $\mathcal{F} T$ can be evaluated instead in $\mathcal{F} T \otimes \mathcal{F} T$, and is equal to

$$
\left((\phi+1)^{-1} \chi_{\widehat{G}}(\phi+1)\right)^{2}=\frac{1}{\phi^{4}}\left(\chi_{\widehat{G}}(\phi+1)\right)^{2} .
$$

Checking two of the three relations is easy. The value for $G=$ circle at $\phi+2$ is $\phi+1$. The corresponding value of $\Psi(G)=G \otimes G$ is $\phi^{2}=\phi+1$. Likewise, tadpoles vanish for any value of $Q$, so the image of the relation on the right in Figure 4 under $\Psi$ clearly holds in $\mathcal{F} T \otimes \mathcal{F} T$.

To derive the H-I relation, consider its image under $\Psi$, shown in Figure 15. Even

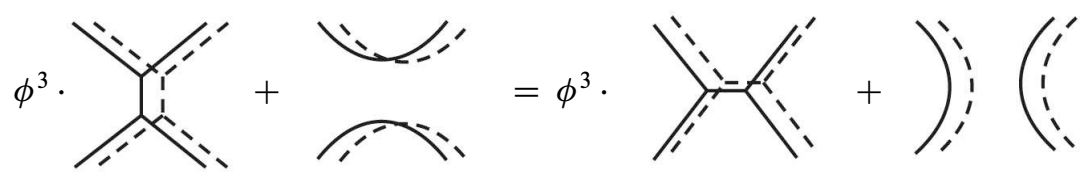

Figure 15: The image under $\Psi$ of the $\mathrm{H}-\mathrm{I}$ relation, defining the chromatic algebra (Figure 4)

though the image of $\mathcal{F} T$ under $\pi_{Q}$ is 1 -dimensional for any value of $Q$, we have 
seen that at $Q=\phi+1$ there is an additional relation (1-2) obeyed by the chromatic polynomial. (We will show in the next section that such additional relations exist for any $Q$ satisfying (1-4).) This relation, established in the previous section, is not a consequence of the deletion-contraction rule, but is still consistent with the projection map $\pi_{\phi+1}$. One checks using the first relation in Figure 4 that (1-3) is equivalent to each of the following two relations (in other words, both of these relations are consistent with the projection $\left.\pi_{\phi+1}\right)$ :

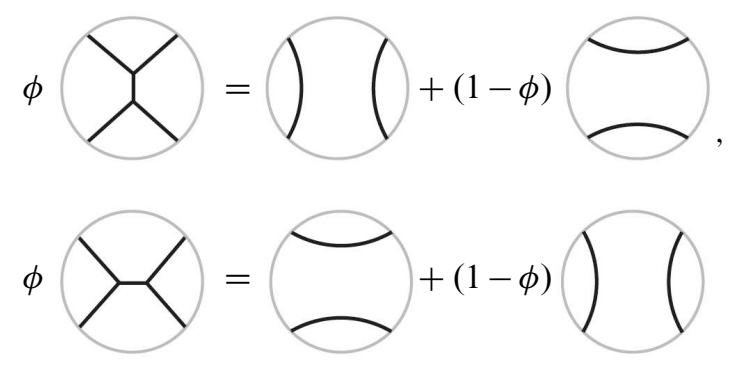

Figure 16: Relations in $\mathcal{C}_{2}^{\phi+1}$, equivalent to Tutte's relation (1-2)

Apply the relation on the left in Figure 16 to the expression in the left hand side in Figure 15, in both copies of $\mathcal{F} T$ at $\phi+1$, and use the identities involving the golden ratio

$$
\phi+1=\phi^{2}, \phi-1=\phi^{-1}
$$

to get the relation in Figure 17.

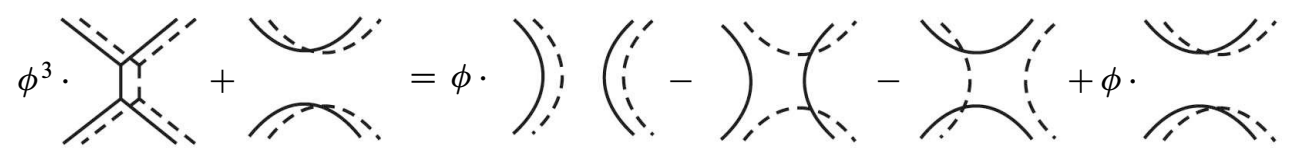

Figure 17

The fact that the expression on the right is invariant under the 90 degree rotation establishes the relation in Figure 15. This concludes the proof of Lemma 3.1 and of Theorem 1.

Remarks (1) The proof of the theorem above could also have been given directly in the context of algebras and their traces. The proof of Lemma 3.1 above shows the existence of a map $\mathcal{C}^{\phi+2} \longrightarrow\left(\mathcal{C}^{\phi+1} / R\right) \otimes\left(\mathcal{C}^{\phi+1} / R\right)$, where $R$ denotes the trace 
radical. One may consider then a diagram involving the Temperley-Lieb algebras, analogous to (2-5), and Theorem 1 follows from applying the trace to these algebras.

(2) The map $\Psi$, considered in the proof of the Theorem 1, has a simple definition (3-3) only in the context of trivalent graphs. The chromatic algebra may be defined in terms of all, not just trivalent, graphs (see the next section), however the extension of $\Psi$ to vertices of higher valence is substantially more involved.

\section{Level-rank duality}

In the previous section we gave an algebraic proof of Tutte's identity (1-1) using the chromatic algebra, without making reference to the Birman-Murakami-Wenzl algebra. In this section we explain how an essential ingredient for a conceptual understanding of this identity is the level-rank duality of the $\mathrm{SO}(N)$ topological quantum field theories and of the BMW algebras. The golden identity (1-1) is a consequence of the duality between the $\mathrm{SO}(3)_{4}$ and $\mathrm{SO}(4)_{3}$ algebras. This viewpoint explains why such an identity relates the chromatic polynomial $\chi(Q)$ at the values $Q=\phi+1, \phi+2$, and why one does not expect a generalization for other values of $Q$.

We will work with $\mathrm{SO}(N)$ Birman-Murakami-Wenzl algebras which underlie the construction of the (doubled) $\mathrm{SO}(N)$ TQFTs (see for example Freedman [9] for a related discussion concerning the TL algebra and the SU(2) theories and Fendley and Krushkal [6, Section 3.1] in the case of BMW(3)). Note that the chromatic algebra and the $\mathrm{SO}(3) \mathrm{BMW}$ algebra are closely related [6].

The $\mathrm{SO}(N)$ BMW algebra $\operatorname{BMW}(N)_{n}$ is the algebra of framed tangles on $n$ strands in $D^{2} \times[0,1]$ modulo regular isotopy and the $\mathrm{SO}(N)$ Kauffman skein relations in Figure $18[3 ; 18]$.

$$
\left.Y-\searrow^{\prime}=\left(q-q^{-1}\right) \| \mid-\circlearrowleft\right), \quad \searrow=q^{1-N}\left|, \quad Y=q^{N-1}\right|
$$

Figure 18

By a tangle we mean a collection of curves (some of them perhaps closed) embedded in $D^{2} \times[0,1]$, with precisely $2 n$ endpoints, $n$ in $D^{2} \times\{0\}$ and $D^{2} \times\{1\}$ each, at the prescribed marked points in the disk. The tangles are framed, ie they are given with a trivialization of their normal bundle. (This is necessary since the last two relations 
in Figure 18 are not invariant under the first Reidemeister move.) As with TL, the multiplication is given by vertical stacking, and we set $\operatorname{BMW}(N)=\bigcup_{n} \operatorname{BMW}(N)_{n}$.

The trace, $\operatorname{tr}_{K}: \operatorname{BMW}(N)_{n} \longrightarrow \mathbb{C}$, is defined on the generators (framed tangles) by connecting the top and bottom endpoints by standard arcs in the complement of $D^{2} \times[0,1]$ in 3-space, sweeping from top to bottom, and computing the $\mathrm{SO}(N)$ Kauffman polynomial (given by the skein relations above) of the resulting link. The skein relations imply that deleting a circle has the effect of multiplying the element of $\operatorname{BMW}(N)$ by

$$
d_{N}=1+\frac{q^{N-1}-q^{-(N-1)}}{q-q^{-1}} .
$$

In fact, it follows from the first relation in Figure 18 that the subalgebra of $\mathrm{BMW}(N)$ generated by $e_{i}=1-\left(B_{i}-B_{i}^{-1}\right) /\left(q-q^{-1}\right)$ is TL algebra $\mathrm{TL}^{d_{N}}$.

A planar version of the $\mathrm{SO}(N)$ BMW algebra, generated by 4-valent planar graphs, is found by using

$$
(\times)=q()()-(\times)+q^{-1}(\asymp)=q^{-1}()()-(入) .+q(\asymp)
$$

When $N=3$, the relations in this planar version reduce to the chromatic algebra relations $[7 ; 6]$.

When $q$ is a root of unity, the BMW algebra has a variety of special properties. A key property for us occurs when the "level" $k$, defined via

$$
q=e^{i \pi /(k+N-2)}
$$

is an integer. The algebra $\operatorname{BMW}(N)$ for $k$ integer (which we label by $\operatorname{BMW}(N, k)$ ) is related to $\mathrm{SO}(N)$ Chern-Simons topological field theory at level $k$ and the WessZumino-Witten conformal field theory with symmetry algebra given by the Kac-Moody algebra $\mathrm{SO}(N)_{k}[28 ; 25]$. Note that with this definition of $k$, we have $q^{N-1}=$ $-q^{-(k-1)}$. It is then easy to check if a braid $B$ obeys the relations in $\operatorname{BMW}(N, k)$, then $-B^{-1}$ obeys $\operatorname{BMW}(k, N)$.

We use this special property to define an algebra homomorphism $\psi: \operatorname{BMW}(N, k) \longrightarrow$ $\operatorname{BMW}(k, N)$, which sends an additive generator (a framed tangle in $D^{2} \times[0,1]$ ) to a tangle with each crossing reversed, and multiplied by $(-1)^{\# \text { crossings }}$. 
Lemma 4.1 The map $\psi$ defined above is an isomorphism, and moreover it preserves the trace:

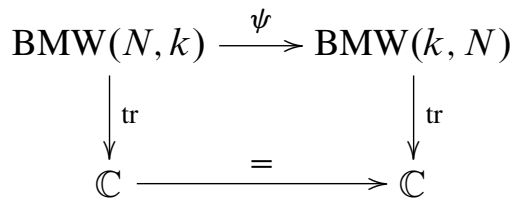

Recall that the traces of the two algebras are the $\mathrm{SO}(N)$, respectively $\mathrm{SO}(k)$, Kauffman polynomials evaluated at $q=e^{i \pi /(k+N-2)}$. The proof of the lemma is checked directly from definitions: $\psi$ is well-defined with respect to the relations in Figure 18 and the last two Reidemeister moves, and $\psi$ has the obvious inverse. The diagram above commutes since computing the trace (the Kauffman polynomial) involves the relations in Figure 18. Note that the weight of a circle $d_{N}$ from (4-1) written in terms of $k$ does not change if $N$ and $k$ are interchanged. This isomorphism is an algebra analogue of level-rank duality (for $\mathrm{SO}(N)$ TQFTs).

The golden identity (1-1) comes from studying the very special case of the duality between $\mathrm{SO}(3)_{4}$ and $\mathrm{SO}(4)_{3}$ theories, where $q=\exp (i \pi / 5)$, and then exploiting a known but possibly underappreciated fact about the SO(4) BMW algebra: it can be decomposed into the product of two TL algebras. This decomposition generalizes the isomorphism between the Lie algebras $\mathrm{SO}(4)$ and $\mathrm{SU}(2) \times \mathrm{SU}(2)$, where the fundamental four-dimensional representation of $\mathrm{SO}(4)$ decomposes into the product of two spin-1/2 representations of $\mathrm{SU}(2)$. The homomorphism $\Psi$ used in the previous section is the chromatic analogue of the composite isomorphism from $\mathrm{SO}(3)_{4}$ to $\mathrm{SO}(3)_{3 / 2} \otimes \mathrm{SO}(3)_{3 / 2}$.

To make this precise, consider the "skein" presentation, TL ${ }^{\text {skein }}$, of the TemperleyLieb algebra, given by framed tangles in $D^{2} \times[0,1]$ modulo regular isotopy and the skein relations defining the Kauffman bracket (see Section 2 in [6]). The map $\mathrm{BMW}(4)_{n}^{q} \rightarrow \mathrm{TL}_{n}^{d} \otimes \mathrm{TL}_{n}^{d}$ takes each generator $g, g=B, B^{-1}$ or $e$ to the product of the corresponding elements in TL ${ }^{\text {skein }}, g \mapsto g \otimes g$. The parameters $q$ and $d$ in these algebras are related by $d=-q-q^{-1}$ as before; note that the weight $d_{4}=\left(q+q^{-1}\right)^{2}$ of a circle in BMW(4) from (4-1) is indeed the square of the weight of a circle in $\mathrm{TL}^{d}$. This map is well-defined: the Reidemeister moves and the BMW relations in Figure 18 hold in TL $\otimes \mathrm{TL}$ as a consequence of the Reidemeister moves and the Kauffman bracket skein relations. The same argument shows that this map preserves the trace. In the planar formulation, the crossing defined by (4-2) is expressed as a linear combination in TL $\otimes \mathrm{TL}$, as displayed in Figure 19. We denote one of the TL copies with a dashed line to emphasize that these copies are independent. 


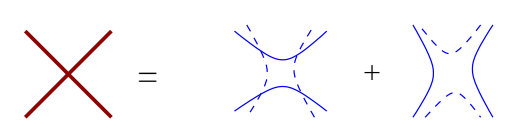

Figure 19: The crossing in BMW(4) mapped to TL $\otimes$ TL

The final ingredient in this proof of Tutte's identity (1-1) is the isomorphism between $\operatorname{BMW}(3,3 / 2) / R$ and TL, where $R$ denotes the trace radical. Using the relation between the SO(3) BMW algebra and the Temperley-Lieb algebra [6], one observes that the relation in Figure 20 is in the trace radical of the (planar presentation of) $\operatorname{BMW}(3,3 / 2)$. (In fact, this relation is equivalent to Tutte's relation (1-2).) Using this relation, $\operatorname{BMW}(3,3 / 2) / R$ is mapped to TL by resolving all $4-$ valent vertices. The inverse map, TL $\longrightarrow$ BMW $(3,3 / 2)$, is given by mapping the generators $e_{i}$ of TL to the same generators of the BMW algebra.

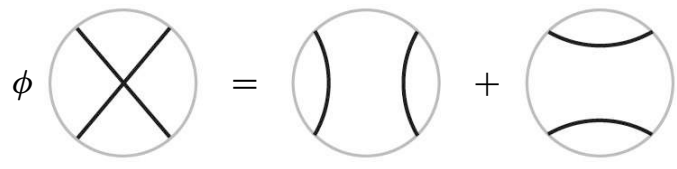

Figure 20

The golden identity follows from the duality between $\operatorname{BMW}(3,4)$ and $\operatorname{BMW}(4,3)$, and then mapping the latter into a product of two copies of $\operatorname{BMW}(3,3 / 2) / R$. The map from BMW(4) $)^{q}$ to $\mathrm{TL}^{d} \otimes \mathrm{TL}^{d}$ above exists for any value of $q$, and at the special value $q=\exp (i \pi / 5)$ we showed above that this map lifts to a homomorphism

$$
\operatorname{BMW}(4,3) \longrightarrow(\operatorname{BMW}(3,3 / 2) / R) \otimes(\operatorname{BMW}(3,3 / 2) / R) .
$$

The trace of the $\mathrm{SO}(3) \mathrm{BMW}$ algebra is given (up to a normalization) by the chromatic polynomial; see Theorem 6.3 and Corollary 6.5 in [6]. Tutte's identity then follows by applying the trace to the algebras above: the trace of $\operatorname{BMW}(4,3) \cong \operatorname{BMW}(3,4)$ is given by the chromatic polynomial evaluated at $Q=\phi+2$, while the product of the traces on the right corresponds to $(\chi(\phi+1))^{2}$.

The fact that the golden identity relates a chromatic polynomial squared to the chromatic polynomial at another value is very special to these values of $Q$. Generalizations using level-rank duality $\mathrm{SO}(N)_{4} \rightarrow \mathrm{SO}(4)_{N}$ for other values of $N$ do not involve the chromatic polynomial. Generalizations using $\mathrm{SO}(N)_{3} \rightarrow \mathrm{SO}(3)_{N}$ at other $N$ give a linear relation between the chromatic polynomial and the Markov trace for the $\mathrm{SO}(N)_{3}$ BMW algebra, very unlike the golden identity. 


\section{Tutte's relations and Beraha numbers}

In this section we establish relations for the chromatic polynomial evaluated at any value of

$$
Q=2+2 \cos \left(\frac{2 \pi j}{n+1}\right),
$$

for any positive integers obeying $j<n$. Each such relation is independent of (but consistent with) the contraction-deletion relation, and Lemmas 5.3 and 5.5 give a recursive formula for them. Our result generalizes Tutte's identity (1-2) to this set of $Q$; note that the values (5-1) are dense in the interval $[0,4]$ in the real line. When $j=1$, these are the Beraha numbers, which strong numerical evidence suggests are the accumulation points of the zeros of $\chi(Q)$ for planar triangulations. Tutte had conjectured such a relation would exist for all Beraha numbers, and found examples in several cases [24]. Our results follow from the observation that the chromatic polynomial relations for a given value of $Q$ correspond to elements of the trace radical of the chromatic algebra $\mathcal{C} Q$. To derive these relations, it is convenient to define the chromatic algebra in terms of all planar graphs, not just the trivalent ones used above.

\subsection{A presentation of the chromatic algebra via the contraction-deletion rule}

Consider the set $\mathcal{G}_{n}$ of the isotopy classes of planar graphs $G$ embedded in the rectangle $R$ with $n$ endpoints at the top and $n$ endpoints at the bottom of the rectangle. (The intersection of $G$ with the boundary of $R$ consists precisely of these $2 n$ points (Figure 3 ) and the isotopy of graphs is required to preserve the boundary.) It is convenient to divide the set of edges of $G$ into outer edges, ie those edges that have an endpoint on the boundary of $R$, and inner edges, whose vertices are in the interior of $R$.

The relations in 2.1 defining the chromatic algebra apply to trivalent graphs. Instead of generalizing them directly, we define the chromatic algebra here using the contractiondeletion rule. It is shown in [6] that the two definitions Definition 2.1 and Definition 5.1) give isomorphic algebras. Analogously to the other definition, the idea is to view the contraction-deletion rule (2-1) as a linear relation between the graphs $G, G / e$ and $G \backslash e$. To make this precise, let $\mathcal{F}_{n}$ denote the free algebra over $\mathbb{C}[Q]$ with free additive generators given by the elements of $\mathcal{G}_{n}$. The multiplication is given by vertical stacking. Define $\mathcal{F}=\bigcup_{n} \mathcal{F}_{n}$. Consider the following set of local relations on the elements of $\mathcal{G}_{n}$. (Note that these relations only apply to inner edges which do not connect to the top and the bottom of the rectangle.) 


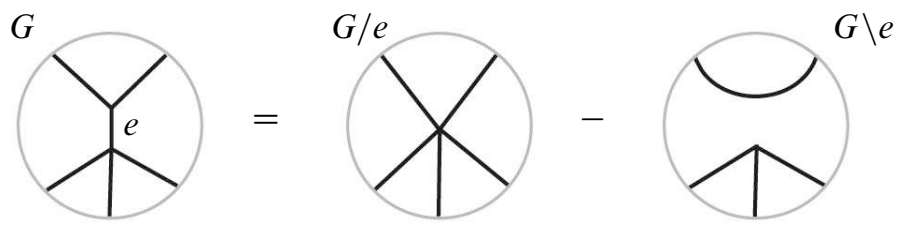

Figure 21: Relation (1) in the chromatic algebra

(1) If $e$ is an inner edge of a graph $G$ which is not a loop, then $G=G / e-G \backslash e$, as illustrated in Figure 21.

(2) If $G$ contains an inner edge $e$ which is a loop, then $G=(Q-1) G \backslash e$, as in Figure 22. (In particular, this relation applies if $e$ is a loop not connected to the rest of the graph.)

(3) If $G$ contains a 1-valent vertex (in the interior of the rectangle) as in Figure 22, then $G=0$.
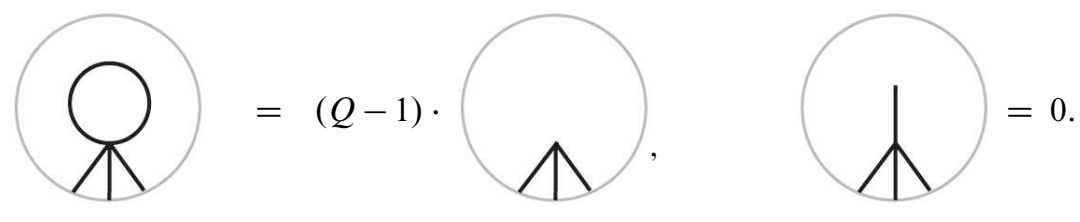

Figure 22: Relations (2) and (3) in the chromatic algebra

Definition 5.1 The chromatic algebra in degree $n, \overline{\mathcal{C}_{n}}$, is an algebra over $\mathbb{C}[Q]$ which is defined as the quotient of the free algebra $\mathcal{F}_{n}$ by the ideal $I_{n}$ generated by the relations (1), (2) and (3). $\overline{\mathcal{C}}_{n}^{Q}$ denotes the algebra over $\mathbb{C}$ when $Q$ is specialized to a complex number. Set $\overline{\mathcal{C}}=\bigcup_{n} \overline{\mathcal{C}}_{n}$. Analogously to Section 2 , the trace, $\operatorname{tr}_{\chi}: \overline{\mathcal{C}} Q \longrightarrow \mathbb{C}$ is defined on the additive generators (graphs) $G$ by connecting the endpoints of $G$ by arcs in the plane (denote the result by $\bar{G}$ ) and evaluating

$$
Q^{-1} \cdot \chi_{\widehat{G}}(Q) \text {. }
$$

One checks that the trace is well-defined with respect to the relations (1)-(3). For example, the relation (1) corresponds to the contraction-deletion rule for the chromatic polynomial of the dual graph: $\chi_{\widehat{G}}=\chi_{\widehat{G} \backslash \widehat{e}}-\chi_{\widehat{G} / \widehat{e}}$, where $\widehat{e}$ is the edge of $\widehat{G}$ dual to $e$. The relation (2) corresponds to deleting a 1 -valent vertex and the adjacent edge of the dual graph, and the chromatic polynomial vanishes in case (3) since the dual graph has a loop. 
Remark The trace may also be described in terms of the flow polynomial of $\bar{G}$. Both the flow polynomial and the chromatic polynomial are one-variable specializations of the two-variable Tutte polynomial [4].

Consider the algebra homomorphism $\bar{\Phi}: \overline{\mathcal{C}}_{n}^{d^{2}} \longrightarrow \mathrm{TL}_{2 n}^{d}$, analogous to the one given in Section 2 for trivalent graphs shown in Figure 23. The factor in the definition of $\bar{\Phi}$ corresponding to a $k$-valent vertex is $d^{(k-2) / 2}$, for example it equals $d$ for the $4-$ valent vertex in Figure 23. The overall factor for a graph $G$ is the product of the factors $d^{(k(V)-2) / 2}$ over all vertices $V$ of $G$.

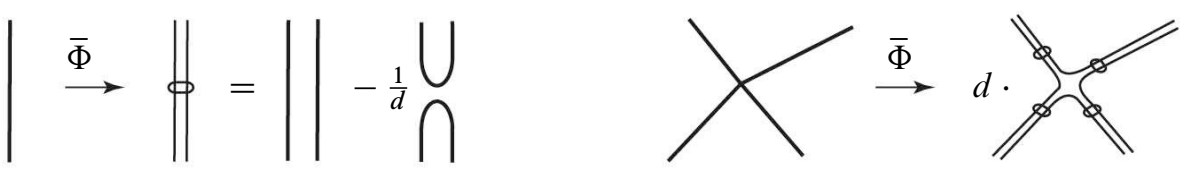

Figure 23: Definition of the homomorphism $\bar{\Phi}: \overline{\mathcal{C}}_{n}^{Q} \longrightarrow \mathrm{TL}_{2 n}^{\sqrt{Q}}$

One checks that $\bar{\Phi}$ is well-defined. For example, for the defining relation (1) of the chromatic algebra in Figure 21, one applies $\bar{\Phi}$ to both sides and expands the projector at the edge $e$, as shown in Figure 23. The resulting relation holds due to the choices of the powers of $d$ corresponding to the valencies of the vertices. Similarly, one checks the relations (2) and (3).

It is shown in [6, Section 4] that the inclusion \{trivalent graphs $\} \subset\{$ all graphs $\}$ induces an isomorphism of the algebras $\mathcal{C}_{n}^{Q}, \overline{\mathcal{C}}_{n}^{Q}$ in Definitions 2.1 and 5.1. Therefore from now on we will often use the same notation, $\mathcal{C}_{n}^{Q}$, for both. It follows that the version of Lemma 2.5 holds for the algebra $\overline{\mathcal{C}}_{n}^{Q}$ defined in Definition 5.1. Specifically, in the notation of Lemma 2.5, for any planar graph $G, Q^{-1} \chi_{Q}(\widehat{G})=\bar{\Phi}(G)$, and therefore $\bar{\Phi}$ preserves the algebra traces:

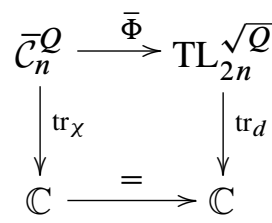

This is true since any graph generator of $\overline{\mathcal{C}}_{n}^{Q}$ in Definition 5.1 is equivalent to a linear combination of trivalent graphs, using the contraction-deletion rule (1). The commutativity of the diagram above then follows from Lemma 2.5 and the fact that $\bar{\Phi}$ and the traces $\operatorname{tr}_{\chi}, \operatorname{tr}_{d}$ of the two algebras are well-defined. 


\subsection{Chromatic polynomial relations and the trace radical}

It follows from (5-2) that the pullback of the trace radical in $\mathrm{TL}_{2 n}^{\sqrt{Q}}$ is in the trace radical of $\mathcal{C}_{n}^{Q}$. (Recall that the trace radical in the algebra $A$, where $A=\mathcal{C}_{n}^{Q}$ or $\mathrm{TL}_{n}^{d}$, is the ideal consisting of the elements $a$ of $A$ such that $\operatorname{tr}(a b)=0$ for all $b \in A$.)

Next observe that the local relations on graphs which preserve the chromatic polynomial of the dual, for a given value of $Q$, correspond to the elements of the trace radical in $\mathcal{C} Q$. (By dualizing the relation, one gets relations which preserve the chromatic polynomial of the graphs themselves, as opposed to that of their dual graphs; for example see figures 1 and 2.) Indeed, suppose $R=\sum_{i} a_{i} G_{i}$ is a relation among graphs in a disk $D$, so each graph $G_{i}$ has the same number of edges meeting the boundary of the disk. Suppose first that this number is even, say equal to $2 n$. Divide the boundary circle of the disk into two intervals, so that each of them contains precisely $n$ endpoints of the edges. Consider the disk $D$ as a subset of the $2-$ sphere $S^{2}$. The fact that $R$ is a relation means that for any fixed graph $G$ in the complement $S^{2} \backslash D$, with the same $2 n$ points on the boundary, the linear combination

$$
\sum_{i} a_{i} \chi \widehat{G_{i} \cup G}
$$

vanishes at $Q$. Since both the disk $D$ and its complement $S^{2} \backslash D$ are homeomorphic to a rectangle, one may consider $R$ as an element in $\mathcal{C}_{n}^{Q}$, and moreover it is in the trace radical: $\operatorname{tr}(R \cdot G)=0$, and the graphs $G$ additively generate $\mathcal{C}_{n}$.

Converting the disk $D$ above into a rectangle, the subdivision of the $2 n$ boundary points into two subsets of $n$, and the fact that this number is even, may seem somewhat artificial. This reflects the algebraic structure of the setting we are working in. The discussion may be carried through in the context of the chromatic category, and further planar algebra, where the algebraic structure is more flexible while the notion of the trace radical is retained. For example, in the category the multiplication (vertical stacking) is complemented by tensor multiplication (horizontal stacking). Describing these structures in further detail would take us outside the scope of the present paper, so instead we refer the interested reader to Freedman [9, Section 2] where the discussion is given in the similar context of the Temperley-Lieb algebra. One observes that a relation preserving the chromatic polynomial, in fact, corresponds to an element of the ideal closure of the trace radical in the chromatic category, not just algebra. (The importance of this distinction will become clear in the following subsections.) The converse argument shows that an element of the trace radical may be viewed as a relation among planar graphs, preserving the chromatic polynomial of the duals. 


\subsection{The trace radical in $\mathrm{TL}_{2 m}^{d}$ and relations in $\mathcal{C}_{m}^{Q}$}

The structure of the trace radical in the Temperley-Lieb algebra is well-understood. In particular, they occur for each special value of $d$ defined by

$$
d=2 \cos \left(\frac{\pi j}{n+1}\right),
$$

where $j$ and $n$ are positive integers obeying $j<n$. When $j$ and $n+1$ are coprime, the trace radical in $\mathrm{TL}^{d}$ is generated by an element $P^{(n)}$ called the Jones-Wenzl projector $[12 ; 27]$. A theorem of Goodman and Wenzl (see the Appendix of Freedman [9]) shows that for values of $d$ other than (5-3), the Temperley-Lieb category does not have any nontrivial proper ideals. We briefly review the basic properties of these projectors below; see Kauffman and Lins [13] for more details and Freedman [9] for a discussion of the trace radical in the context of the Temperley-Lieb category.

The Jones-Wenzl projector $P_{i}^{(r)}$ acts in $\mathrm{TL}_{n}$ for any value of $d$ with $n \geq r$; when $n>r$ we include the subscript to indicate that it is acting nontrivially on the strands labeled $i, i+1, \ldots, i+r-1$. The first two Jones-Wenzl projectors are $P^{(1)}=1$, and $P_{i}^{(2)}=1-E_{i}$. (We use the notation for the generators of the Temperley-Lieb algebra introduced in Section 2.2.) A recursive formula (cf [13]) giving the rest is

$$
P^{(n)}=P_{1}^{(n-1)}-\frac{d \Delta_{n-2}}{\Delta_{n-1}} P_{1}^{(n-1)} E_{n-1} P_{1}^{(n-1)},
$$

where the number $\Delta_{n}$ is simply the trace:

$$
\Delta_{n}=\operatorname{tr}_{d} P^{(n)}
$$

This recursion relation is illustrated in Figure 24. Taking the trace of the elements in

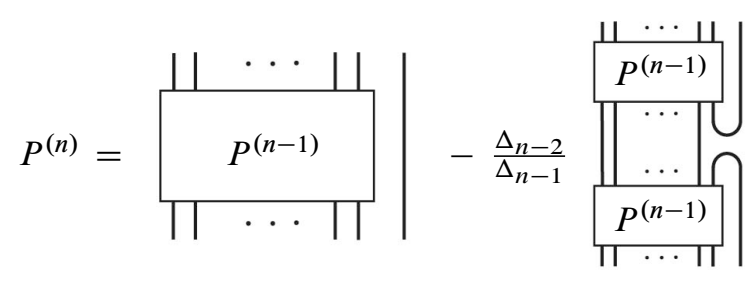

Figure 24: A recursive formula for the Jones-Wenzl projectors in the Temperley-Lieb algebra

the recursion relation yields $\Delta_{n}=d \Delta_{n-1}-\Delta_{n-2}$. Since $\Delta_{1}=d$ and $\Delta_{2}=d^{2}-1$,

$$
\Delta_{n}=\frac{\sin ((n+1) \theta)}{\sin (\theta)}
$$


where $\theta$ is defined via $d=2 \cos (\theta)$.

Of course any element in the trace radical has its own trace equal to zero. From the explicit formula (5-6) for $\Delta_{n}$, it follows that $P^{(n)}$ can be in the trace radical only when $\theta=\pi j /(n+1)$, where $j$ is a nonzero integer not a multiple of $n+1$. In terms of $d$, these correspond to the values in (5-3). Indeed, for these values of $d, P^{(n)}$ generates the trace radical, and moreover the theorem of Goodman-Wenzl guarantees that this is the unique proper ideal in the category for coprime $j$ and $n+1$.

Remark A useful way of understanding the Jones-Wenzl projectors is to think of each strand in the Temperley-Lieb algebra as carrying spin-1/2 of the quantum-group algebra $U_{q}(\operatorname{sl}(2))$, where $d=q+q^{-1}$. There exist spin $-r / 2$ representations of this algebra behaving similarly to those of ordinary $\operatorname{su}(2)$, except for the fact at the special values of $d$ in (5-3) they are irreducible only for $r<n$. In the algebraic language, the recursion relation describes taking a tensor product of representations, so that $P^{(r)}$ is the projector onto the largest possible value of spin $r / 2$ possible for $r$ strands. For example, $P_{i}^{(2)}$ projects the strands $i, i+1$ onto spin 1 , while the orthogonal projector $E_{i}$ projects these two strands onto spin 0 . The fact that the spin $-n / 2$ representation at a special value of $d$ is reducible is the reason the corresponding projector $P^{(n)}$ can be set to zero. The strands in the chromatic algebra can be viewed as carrying spin 1 . It is logical to expect that a projector from $m$ spin- 1 strands onto spin $m$ exists, and that it generates a proper ideal. We will show that this is indeed so in the following.

Lemma 5.2 The Jones-Wenzl projector $P^{(2 m)} \in \mathrm{TL}_{2 m}$ is in the image of the chromatic algebra: $P^{(2 m)} \in \Phi\left(\mathcal{C}_{m}\right)$, so its pullback at the corresponding special value of $Q$ is in the trace radical of the chromatic algebra.

Proof Any element $\mathcal{E}$ of $\mathrm{TL}_{n}$ obeying $E_{j} \mathcal{E}=\mathcal{E} E_{j}=0$ for all $j<n$ is said to be "killed by turnbacks". It follows that any such element obeys the equality $P_{j}^{(2)} \mathcal{E} P_{j}^{(2)}=\mathcal{E}$. As is straightforward to check using the recursion relation, the JonesWenzl projectors are killed by turnbacks, as illustrated in the left of Figure 25. Thus

$$
P^{(2 m)}=P_{1}^{(2)} P_{3}^{(2)} \ldots P_{2 m-1}^{(2)} P^{(2 m)} P_{1}^{(2)} P_{3}^{(2)} \ldots P_{2 m-1}^{(2)} .
$$

So we can pair up the strands above and below the Jones-Wenzl projector, and replace each pair with $P_{j}^{(2)}$, as illustrated on the right of Figure 25 .

Consider the projector $P^{(2 m)}$ as a linear combination of curve diagrams in the rectangle. Each pair of external strands of $P^{(2 m)}$ is replaced with $P^{(2)}$, so all external strands in the Jones-Wenzl projector in $\mathrm{TL}_{2 m}$ correspond to lines in the chromatic algebra. Since each diagram (additive generator of the Temperley-Lieb algebra) consists of disjoint 

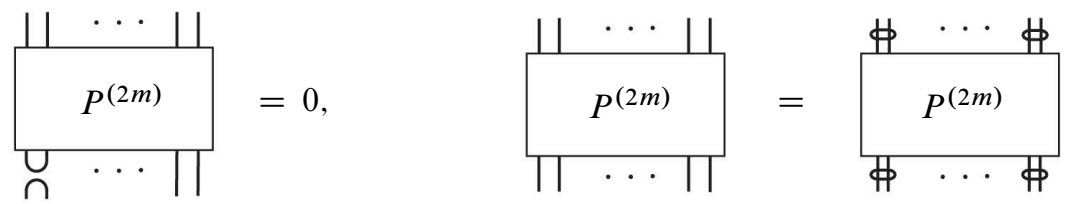

Figure 25

embedded curves, it follows from the definition of $\Phi$ (Figure 23) that each individual term in the expansion of $P^{(2 m)}$, with external strands paired up and replaced with $P^{(2)}$, is in the image of $\Phi$, see Figure 26 for an example. Thus the linear combination, $P^{(2 m)} \in \mathrm{TL}_{2 m}$, is indeed in the image of the chromatic algebra $\mathcal{C}_{m}$.

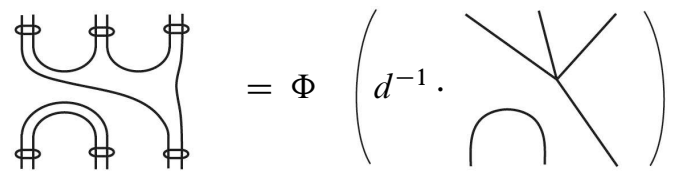

Figure 26

Applying the recursive formula for the Jones-Wenzl projector (Figure 24) twice and using the technique in the proof of Lemma 5.2, one gets the formula for the preimage of $P^{(2 m)}$ in the chromatic algebra:

Lemma 5.3 The pullback $\bar{P}^{(2 m)}$ of the Jones-Wenzl projector $P^{(2 m)}$ to $\mathcal{C}_{m}$ is given by the recursive formula in Figure 27.

The base of this recursion is $\bar{P}^{(2)}$ which is now just a single strand. As mentioned above, the commutativity of diagram (5-2) implies that the pullback of the trace radical in $\operatorname{TL}_{2 m}^{\sqrt{Q}}$ is in the trace radical of $\mathcal{C}_{m}^{Q}$. Therefore Lemma 5.3 establishes a chromatic polynomial identity generalizing (1-2) for each value of $Q$ obeying

$$
Q=4 \cos ^{2}\left(\frac{\pi j}{2 m+1}\right)=2+2 \cos \left(\frac{2 \pi j}{2 m+1}\right)
$$

with $j<2 m$. This generalized identity may be generated explicitly by using the recursion relation for $\bar{P}^{(2 m)}$ in Figure 27. Specifically, the relation $\bar{P}^{(2 m)}=0$ preserves the chromatic polynomial of the dual graphs, and replacing each graph in the relation $\bar{P}^{(2 m)}=0$ by its dual gives a generalization of Tutte's relation (1-2). For example, using the recursive formula shows that (1-3) (checked directly in Section 2 by showing $\Phi$ maps it to $P^{(4)}$ ) is equivalent in the chromatic algebra to setting $\bar{P}^{(4)}=0$. 


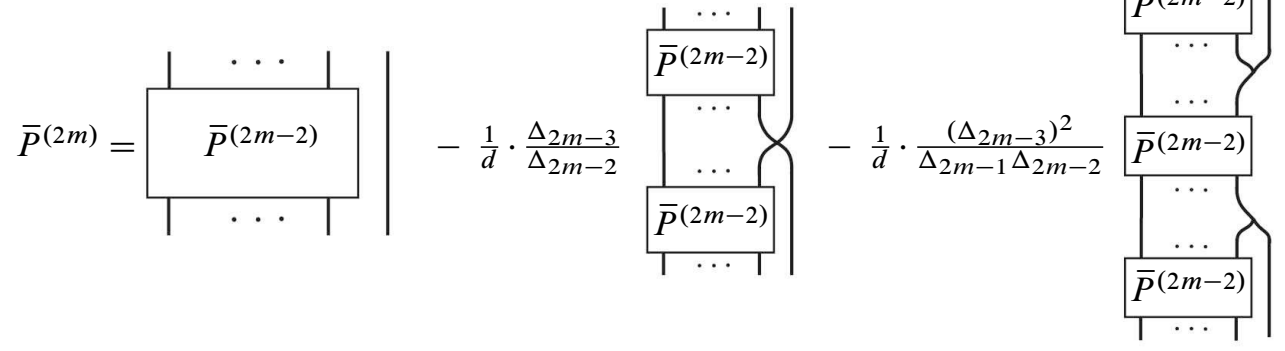

Figure 27: A recursive formula for the pullback $\bar{P}^{(2 m)}$ of the Jones-Wenzl projector $P^{(2 m)}$ in the chromatic algebra

\subsection{Other values of $Q$}

We have used the Jones-Wenzl projectors labeled by even integers to find chromatic identities for half the values of $Q$ in (5-1). In this subsection, we show how to use the projectors $P^{(2 m-1)}$ to find chromatic identities valid at $Q$ in (5-1) for odd $n$.

We start by indicating a direct generalization of the argument above, starting with the Jones-Wenzl projector $P^{(2 m-1)} \in \mathrm{TL}_{2 m-1}$ and getting a relation in $\mathcal{C}_{m}$ at the corresponding value of $Q$. The drawback of this approach is that it involves a choice of including $P^{(2 m-1)}$ into $\mathrm{TL}_{2 m}$. Further below we pass from algebraic to categorical setting to get a unique chromatic relation corresponding to $P^{(2 m-1)}$.

To find chromatic identities for even $n$, we paired up the lines in $P^{(2 m)} \in \mathrm{TL}_{2 m}$ and showed that this projector could be pulled back to the chromatic algebra. Since $P^{(2 m-1)} \in \mathrm{TL}_{2 m-1}$ acts on odd number of lines, finding the generalization of Lemma 5.2 requires a little more work. The simplest way is to map $P^{(2 m-1)}$ to an element in $\mathrm{TL}_{2 m}$ by adding a nonintersecting strand at the right; we label this as $P_{1}^{(2 m-1)} \in \mathrm{TL}_{2 m}$ (see Figure 28). $P_{1}^{(2 m-1)}$ is killed by turnbacks $E_{j}$ for $j<2 m-1$, but to be able to pair up all $2 m$ lines, we need to define the element $R^{(2 m-1)} \in \mathrm{TL}_{2 n}$ as

$$
\begin{aligned}
R^{(2 m-1)} & \equiv P_{2 m-1}^{(2)} P_{1}^{(2 m-1)} P_{2 m-1}^{(2)} \\
& =P_{1}^{(2)} P_{3}^{(2)} \ldots P_{2 m-1}^{(2)} P_{1}^{(2 m-1)} P_{1}^{(2)} P_{3}^{(2)} \ldots P_{2 m-1}^{(2)} .
\end{aligned}
$$

Again see Figure 28. $R$ is no longer a projector, but is killed by all turnbacks with $j \leq 2 m-1$. Therefore we can pair up the strands as before, and a rerun of the argument proving the previous lemma shows that: 

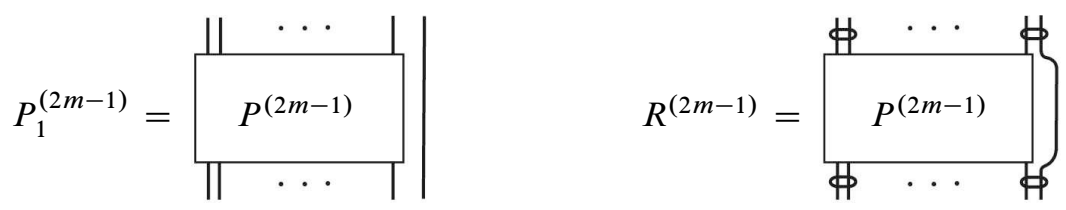

Figure 28

Lemma 5.4 The element $R^{(2 m-1)} \in \mathrm{TL}_{2 m}$ is in the image of the chromatic algebra: $R^{(2 m-1)} \in \Phi\left(\mathcal{C}_{m}\right)$, so its pullback at the corresponding value of $Q$ is in the trace radical of the chromatic algebra.

$R^{(2 m-1)}$ is in the ideal generated by $P^{(2 m-1)}$, thus it is in the trace radical when $Q=d^{2}$ obeys (5-1) for odd $n=2 m-1$. A chromatic identity then follows by taking the sum of the dual graphs of the pictures for the pullback $\Phi^{-1}\left(R^{(2 m-1)}\right)$.

This construction of an ideal in $\mathcal{C}_{m}$ at $\sqrt{Q}=2 \cos (\pi /(2 m))$ is not unique. Other elements of $\mathcal{C}_{m}$ at this value of $Q$ can be set to zero as well, although it is not clear to us if any of these result in new chromatic identities, or simply rotations of each other.

In order to pull back $P^{(2 m-1)}$ to $\mathcal{C}_{m}$, we needed to add an extra line so that we could form $m$ pairs on the bottom and $m$ on the top. To pull back $P^{(2 m-1)}$ itself, and thus to find a unique chromatic identity, we do not add an extra line, but instead pair up a line from the top of $P^{(2 m-1)}$ with one from the bottom. The pullback of this object no longer lives in the chromatic algebra, but rather the chromatic category, briefly discussed at the end of Section 5.2. The three equivalence relations (including the contraction-deletion rule) (1)-(3) at the beginning of Section 5.1 serve as defining relations in this category as well. Finding this pullback results in a chromatic identity involving the duals of graphs on a disk with $2 m-1$ external strands in total. To be more precise, we will consider graphs in a disk $D$ with $n$ fixed points on the boundary of $D$, modulo the relations (1)-(3) in Section 5.1. Moreover, the marked points on the boundary are numbered 1 through $n$. In the categorical language, these are morphisms: elements of $\operatorname{Hom}(0, n)$. Given two such graphs $a, b$, their inner product is computed by reflecting $b$ and gluing the two disks so the numbered points on the boundary are matched. Then $\langle a, b\rangle$ is given by the evaluation of the resulting graph in the sphere (equal to $Q^{-1}$ times the chromatic polynomial of the dual graph). In this setting, the trace radical is replaced by the ideal of negligible morphisms; see Freedman [9] for more details. We extend the map $\Phi$ to the chromatic category (with values in the Temperley-Lieb category) in the obvious way: it is defined by replacing each line and vertex by the doubled lines in TL, as illustrated in Figure 8 and Figure 23. 
We start by showing when the Jones-Wenzl projectors are killed by "end turnbacks". An end turnback on the right in $\mathrm{TL}_{n}$ is the partial trace $\operatorname{tr}_{n}$ : $\mathrm{TL}_{n} \rightarrow \mathrm{TL}_{n-1}$, defined by connecting just the $n$-th strand on the bottom to the $n$-th strand on the top, Figure 29 (left). Likewise, an end turnback on the left is the partial trace $\operatorname{tr}_{1}$. From the recursion relation pictured in Figure 24, we find

$$
\operatorname{tr}_{n}\left(P^{(n)}\right)=\left(d-\frac{\Delta_{n-2}}{\Delta_{n-1}}\right) P^{(n-1)}=\frac{\Delta_{n}}{\Delta_{n-1}} P^{(n-1)} .
$$

When $d$ takes on the special values (5-3), $\Delta_{n}=0$, so only at these values is $P^{(n)}$ killed by the end turnback.
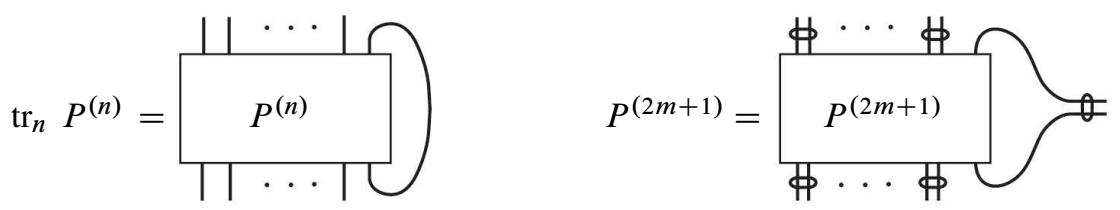

Figure 29: The identity on the right holds in the Temperley-Lieb category at $d=2 \cos (\pi j /(2 m+2))$.

When $P^{(n)}$ is killed by all turnbacks including the end one, it can be pulled back to the chromatic category for any $n$, odd or even. The idea is the same as for the algebras. We pair up the lines in the Temperley-Lieb category, and then replace each pair with a single line in the chromatic category. Figure 29 (right) illustrates the odd case $n=2 m+1$. Note that while Lemma 5.2 is true for any value of $Q$, this categorical analogue for $n=2 m+1$ holds only at the special value of $Q=2+2 \cos (2 \pi j /(2 m+2))$ (and the corresponding value of the parameter $d=\sqrt{Q})$ !

Considering the recursive formula (Figure 24) for the Jones-Wenzl projector $P^{(2 m+1)}$, one gets the following formula for its pullback:

Lemma 5.5 The pullback $\bar{P}^{(2 m+1)}$ of the Jones-Wenzl projector $P^{(2 m+1)}$ to the chromatic category is given by the recursive formula in Figure 30.

Together with Lemma 5.3, this gives a recursive formula for the pullback $\bar{P}^{(n)}$ of the Jones-Wenzl projector $P^{(n)}$ for all values of $n$. Considering the dual graphs for the graphs in the relation $\bar{P}^{(n)}=0$, one gets a chromatic polynomial relation for each value of $Q$ in (5-1).

To give an example, $\bar{P}^{(3)}$ simply is a trivalent vertex. This indeed is in the trace radical when $Q=2$, as is easy to see by reverting to the original definition of the chromatic 


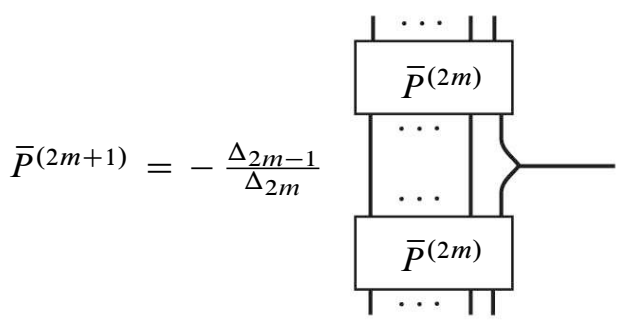

Figure 30: A recursive formula for the pullback $\bar{P}^{(2 m+1)}$ of the Jones-Wenzl projector $P^{(2 m+1)}$ in the chromatic category

polynomial: the dual graph of a trivalent vertex is a triangle, and any graph containing a triangle cannot be colored with two colors.

Acknowledgments We would like to thank Mike Freedman and Kevin Walker for discussions on the Temperley-Lieb category and related questions in quantum topology, as well as for introducing us to the golden identity.

Some of the diagrams in this paper were drawn using Dror Bar-Natan's TeX package dbnsymb [1].

Paul Fendley was supported in part by NSF grants DMR-0412956 and DMR/MSPA0704666, and by the UK EPSRC under grant EP/F008880/1. Vyacheslav Krushkal was supported in part by NSF grants DMS-0605280 and DMS-0729032, and by Harvard University.

\section{References}

[1] D Bar-Natan, dbnsymb (2008) Available at http://www.math.toronto.edu/ $\sim$ drorbn/projects/dbnsymb/dbnsymbman.html

[2] S Beraha, Infinite nontrivial families of maps and chromials, $\mathrm{PhD}$ thesis, Johns Hopkins University (1975)

[3] J S Birman, H Wenzl, Braids, link polynomials and a new algebra, Trans. Amer. Math. Soc. 313 (1989) 249-273 MR992598

[4] B Bollobás, Modern graph theory, Graduate Texts in Math. 184, Springer, New York (1998) MR1633290

[5] P Fendley, Topological order from quantum loops and nets, Annals of Physics 323 (2008) 3113-3136 
[6] P Fendley, V Krushkal, Link invariants, the chromatic polynomial and the Potts model arXiv:0806.3484

[7] P Fendley, N Read, Exact $S$-matrices for supersymmetric sigma models and the Potts model, J. Phys. A 35 (2002) 10675-10704 MR1959217

[8] L Fidkowski, M H Freedman, C Nayak, K Walker, Z Wang, From string nets to nonabelions arXiv:cond-mat/0610583

[9] M H Freedman, A magnetic model with a possible Chern-Simons phase, Comm. Math. Phys. 234 (2003) 129-183 MR1961959 With an appendix by F Goodman and H Wenzl

[10] F Jaeger, On some graph invariants related to the Kauffman polynomial, from: "Progress in knot theory and related topics", Travaux en Cours 56, Hermann, Paris (1997) 69-82 MR1603130

[11] V F R Jones, Planar algebras arXiv:math/9909027

[12] V F R Jones, Index for subfactors, Invent. Math. 72 (1983) 1-25 MR696688

[13] L H Kauffman, SL Lins, Temperley-Lieb recoupling theory and invariants of 3-manifolds, Annals of Math. Studies 134, Princeton University Press (1994) MR1280463

[14] L Kauffmann, H Saleur, An algebraic approach to the planar coloring problem, Comm. Math. Phys. 152 (1993) 565-590 MR1213302

[15] G Kuperberg, Spiders for rank 2 Lie algebras, Comm. Math. Phys. 180 (1996) 109151 MR1403861

[16] P Martin, D Woodcock, The partition algebras and a new deformation of the Schur algebras, J. Algebra 203 (1998) 91-124 MR1620713

[17] E J Mlawer, S G Naculich, H A Riggs, HJ Schnitzer, Group-level duality of WZW fusion coefficients and Chern-Simons link observables, Nuclear Phys. B 352 (1991) 863-896 MR1098252

[18] J Murakami, The Kauffman polynomial of links and representation theory, Osaka J. Math. 24 (1987) 745-758 MR927059

[19] R Penrose, Applications of negative dimensional tensors, from: "Combinatorial Mathematics and its Applications (Proc. Conf., Oxford, 1969)”, Academic Press, London (1971) 221-244 MR0281657

[20] H Saleur, Zeroes of chromatic polynomials: a new approach to Beraha conjecture using quantum groups, Comm. Math. Phys. 132 (1990) 657-679 MR1069841

[21] H N V Temperley, E H Lieb, Relations between the "percolation" and "colouring" problem and other graph-theoretical problems associated with regular planar lattices: some exact results for the "percolation” problem, Proc. Roy. Soc. London Ser. A 322 (1971) 251-280 MR0498284 
[22] W T Tutte, More about chromatic polynomials and the golden ratio, from: "Combinatorial Structures and their Applications (Proc. Calgary Internat. Conf., 1969)", Gordon and Breach, New York (1970) 439-453 MR0263698

[23] W T Tutte, On chromatic polynomials and the golden ratio, J. Combinatorial Theory 9 (1970) 289-296 MR0272676

[24] W T Tutte, Graph theory as I have known it, Oxford Lecture Ser. in Math. and its Appl. 11, The Clarendon Press, Oxford University Press, New York (1998) MR1635397 With a foreword by U S R Murty

[25] K Walker, On Witten's 3-manifold invariants Available at http://canyon23.net/ math/1991TQFTNotes.pdf

[26] K Walker, Talk given at IPAM conference "Topological Quantum Computing”, Technical report (2007) Available at https://www.ipam.ucla.edu/publications/ tqc2007/tqc2007_6544.pdf

[27] H Wenzl, On sequences of projections, C. R. Math. Rep. Acad. Sci. Canada 9 (1987) 5-9 MR873400

[28] E Witten, Quantum field theory and the Jones polynomial, Comm. Math. Phys. 121 (1989) 351-399 MR990772

[29] S Yamada, An operator on regular isotopy invariants of link diagrams, Topology 28 (1989) 369-377 MR1014466

PF: All Souls College and the Rudolf Peierls Centre for Theoretical Physics, University of Oxford

1 Keble Road, Oxford OX13NP, UK

PF, VK: Department of Mathematics, University of Virginia

Charlottesville, VA 22904-4137, USA

fendley@virginia.edu, krushkal@virginia.edu

Proposed: Joan Birman

Seconded: Dave Gabai, Danny Calegari
Received: 31 July 2008 Accepted: 6 November 2008 